OPEN ACCESS

Edited by:

Zhenyu Li,

University of Science and Technology of China, China

Reviewed by:

Xiaojun Wu,

University of Science and Technology of China, China

Liang Chen,

Chinese Academy of Sciences, China Jun-Wei Luo,

Chinese Academy of Sciences, China

*Correspondence:

Ghanshyam Pilania gpilania@lanl.gov

Specialty section:

This article was submitted to Computational Materials Science,

a section of the journal

Frontiers in Materials

Received: 29 February 2016 Accepted: 06 April 2016 Published: 26 April 2016

Citation:

Pilania G, Balachandran PV, Kim C and Lookman T (2016) Finding New

Perovskite Halides via Machine

Learning.

Front. Mater. 3:19

doi: 10.3389/fmats.2016.00019

\section{Finding New Perovskite Halides via Machine Learning}

\author{
Ghanshyam Pilania ${ }^{1 *}$, Prasanna V. Balachandran ${ }^{2}$, Chiho Kim $^{3}$ and Turab Lookman ${ }^{2}$ \\ ${ }^{1}$ Materials Science and Technology Division, Los Alamos National Laboratory, Los Alamos, NM, USA, ${ }^{2}$ Theoretical Division, \\ Los Alamos National Laboratory, Los Alamos, NM, USA, ${ }^{3}$ Department of Materials Science and Engineering, Institute of \\ Materials Science, University of Connecticut, Storrs, CT, USA
}

Advanced materials with improved properties have the potential to fuel future technological advancements. However, identification and discovery of these optimal materials for a specific application is a non-trivial task, because of the vastness of the chemical search space with enormous compositional and configurational degrees of freedom. Materials informatics provides an efficient approach toward rational design of new materials, via learning from known data to make decisions on new and previously unexplored compounds in an accelerated manner. Here, we demonstrate the power and utility of such statistical learning (or machine learning, henceforth referred to as $\mathrm{ML}$ ) via building a support vector machine (SVM) based classifier that uses elemental features (or descriptors) to predict the formability of a given $\mathrm{ABX}_{3}$ halide composition (where $\mathrm{A}$ and $\mathrm{B}$ represent monovalent and divalent cations, respectively, and $\mathrm{X}$ is $\mathrm{F}, \mathrm{Cl}, \mathrm{Br}$, or I anion) in the perovskite crystal structure. The classification model is built by learning from a dataset of 185 experimentally known $\mathrm{ABX}_{3}$ compounds. After exploring a wide range of features, we identify ionic radii, tolerance factor, and octahedral factor to be the most important factors for the classification, suggesting that steric and geometric packing effects govern the stability of these halides. The trained and validated models then predict, with a high degree of confidence, several novel $\mathrm{ABX}_{3}$ compositions with perovskite crystal structure.

Keywords: perovskites, informatics, support vector machines, formability, materials discovery

\section{INTRODUCTION}

The materials community is currently witnessing a fundamental change in the way novel materials are designed and discovered. A steady increase in computational power, accompanied by developments in quantum theory and algorithmic breakthroughs that allow for efficient yet accurate quantum mechanical computations, opens the door to computing properties of a wide range of materials that once seemed prohibitively expensive. As a result, high-throughput explorations of the vast chemical space are increasingly being pursued and have significantly aided our intuition and knowledge-base of material properties (Ceder et al., 2011; Jain et al., 2011; Yu and Zunger, 2012; Curtarolo et al., 2013; Pilania et al., 2013, 2016; Sharma et al., 2014; Balachandran et al., 2016; Kim et al., 2016; Mannodi-Kanakkithodi et al., 2016). Massive open source databases of materials properties (including electronic structure, thermodynamic, and structural properties) are now available on the web (Curtarolo et al., 2012; Computational Materials Repository, 2015; Materials Project - A Materials Genome Approach, 2015). Big-data materials infrastructure (Service, 2012) is increasingly being built with the intent of knowledge extraction and rule-mining to identify candidate materials for next-generation materials breakthroughs. 
To illustrate the efficacy and utility of the informatics route to materials discovery, we here take-up a specific example of predicting the formability of perovskite halides - a class of technologically relevant materials (Mitchell, 2002) possessing a number of interesting properties, including high resistivity and breakdown field, electron-acceptor behavior, a large optical transmission domain, photoluminescence, ionic conductivity over a wide temperature range, antiferromagnetism, ferroelectricity, and piezoelectricity (Muller and Roy, 1974; Sarukura et al., 2007; Zhang et al., 2008; Pilania and Lookman, 2014; Pilania and Uberuaga, 2015).

A typical cubic crystal structure adopted by $\mathrm{ABX}_{3}$ perovskite halides [with three-dimensional arrangement of corner-sharing octahedral $\mathrm{BX}_{6}$ units (Muller and Roy, 1974)] is depicted in Figure 1A, where $A$ and $B$ cations are 12- and 6-fold coordinated, and have $+1,+2$ nominal charge states, respectively, while $\mathrm{X} \in\{\mathrm{F}, \mathrm{Cl}, \mathrm{Br}, \mathrm{I}\}$ represents a halide. However, non-perovskite structures with edge sharing octahedral arrangement are also common in compounds with $\mathrm{ABX}_{3}$ stoichiometry (for instance, $\mathrm{CsNiF}_{3}$ and $\mathrm{CsCoCl}_{3}$ compounds) (Muller and Roy, 1974). The central focus of this paper is a basic task: from available data on formability of $\mathrm{ABX}_{3}$ solids (i.e., known compounds with perovskite or non-perovskite labels), can we construct a ML model and predict with a high degree of accuracy whether a proposed solid with given choices of $\mathrm{A}, \mathrm{B}$, and $\mathrm{X}$ should be a perovskite or a non-perovskite?

Given the technological importance of perovskites, formability of both oxides (Li et al., 2004; Zhang et al., 2007; Feng et al., 2008; Kumar et al., 2008) and halides (Li et al., 2008) falling in this class has been previously studied. These studies performed a classification into perovskite or non-perovskite in the traditional way by using a structure map. A structure map is defined as a two-dimensional plot of the values of two features of the known solids and with lines drawn using ad hoc principles (including by hand) that separate the data points into the different classes of crystal structures (Mooser and Pearson, 1959). The tolerance and octahedral factors are the two most widely used structure governing features in these plots (Li et al., 2004, 2008; Zhang et al., 2007; Feng et al., 2008; Kumar et al., 2008). Our work is a departure from these earlier publications: we consider a large number of potential features that are known to govern the crystal structures of inorganic solids (beyond tolerance and octahedral factors), utilize state-of-the-art ML methods to rationally establish the decision boundaries (based on available data and cross-validation methods) that separate perovskites from non-perovskites and accurately quantify prediction accuracies. By exploring a vast number of different feature combinations, we identify new classifiers, previously unknown, that complement (or could even potentially substitute) the two popular geometric factors used in the past.

While drawing that the standard structure map is not possible with more than two features, ML provides an alternative, more rigorous, and automated method of classification. Unlike the traditional approach adopted for structure maps, where decision boundaries are drawn by hand, in the ML approach model parameters govern the position of decision boundaries and optimal parameters are selected by evaluating the model performance (or prediction accuracy) on unseen data (Pilania et al., 2015a,b).

For ML, we used the support vector classification (SVC) method, which is commonly used in binary classification problems (Vapnik, 1995, 1998; Flach, 2012). An additional advantage of using this method is that besides returning a classification model, it also provides probabilistic estimates of confidence in those predictions that can be very useful in forecasting new candidates, which are yet to be synthesized. In fact, after performing training and testing steps on the available 185 compounds, we employ the best performing model to predict formability of 455 $\mathrm{ABX}_{3}$ chemistries falling within the same chemical space spanned by the training data. The top 20 new $\mathrm{ABX}_{3}$ compounds with high prediction probability of forming perovskite-type crystal structure are, thus, identified. In what follows, we describe the details of our study.

\section{DATASET, FEATURES, AND CHEMICAL SPACE}

This section describes the details of features and perovskite halide formability dataset that were used to train and test the prediction performance of the ML models developed here. Besides the tolerance and octahedral factor feature pairs, we also considered the $\mathrm{A}, \mathrm{B}$, and $\mathrm{X}$ ionic radii (denoted as $r_{A}^{i}, r_{B}^{i}$, and $r_{X}^{i}$, respectively), the bond valence distances of $\mathrm{A}$ and $\mathrm{B}$ from $\mathrm{X}$ (denoted as $r_{A-X}^{b}$ and $r_{B-X}^{b}$ ) (Zhang et al., 2007), and the ratio of the sum of the $s$ and $p$ orbital radii of the $\mathrm{A}$ and $\mathrm{B}$ atoms relative to that of the $\mathrm{X}$ atom (i.e., $r_{A}^{s+p} / r_{X}^{s+p}$ and $r_{B}^{s+p} / r_{X}^{s+p}$ ) (Rabe et al., 1992). Finally, differences in the Martynov-Batsanov electronegativity scales of $\mathrm{A}-\mathrm{X}$ and $\mathrm{B}-\mathrm{X}$ atoms pairs (i.e., $\Delta \chi_{A-X}^{M B}$ and $\Delta \chi_{B-X}^{M B}$ ) were also included. Initial tests, however, showed that these last two features when multiplied, respectively, by the ionic radii ratios $r_{A}^{i} / r_{X}^{i}$

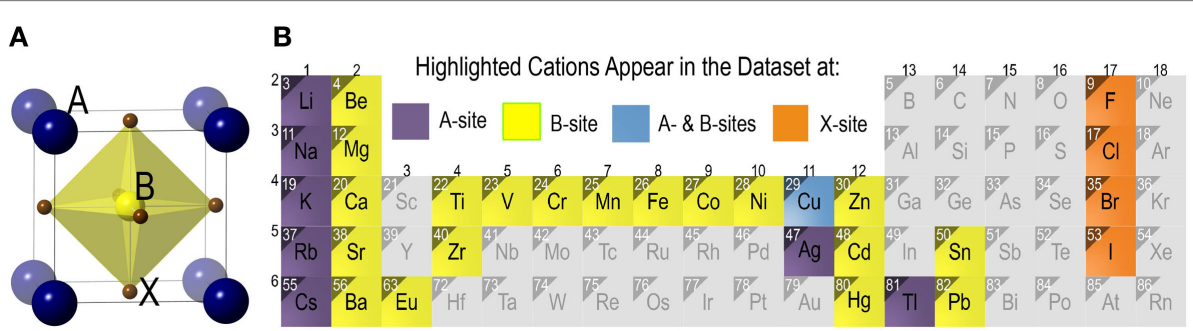

FIGURE 1 | (A) The prototypical cubic perovskite halide crystal structure of $A B X_{3}$ compounds with corner sharing octahedra of halide anions $(X)$ housing $B$-site cations. A-site cations occupy a 12-fold coordination site surrounding $\mathrm{BX}_{6}$ octahedra (shown explicitly in the figure). (B) Chemical space of the $\mathrm{ABX}_{3}$ halide chemistries explored in the present study. Cations appearing at the A-site and/or the B-site are highlighted. $\mathrm{X}$ site can be occupied by four halides in group 17. 
and $r_{B}^{i} / r_{X}^{i}$ perform slightly better and, therefore, $\left(r_{A}^{i} / r_{X}^{i}\right) \Delta \chi_{A-X}^{M B}$ and $\left(r_{B}^{i} / r_{X}^{i}\right) \Delta \chi_{B-X}^{M B}$ were included as features instead of just the differences in the Martynov-Batsanov electronegativity scales of A-X and B-X atoms pairs. Rabe et al. (1992) have shown that the pseudopotential core radii sum and Martynov-Batsanov electronegativity are widely transferable across crystal classes and capture important trends essential to describe the electronic charge distribution, crystal geometry, and bond lengths. We explore the relevance of these features for classifying perovskite and nonperovskite halides.

Bond valence radii that we have used in this work refers particularly to the bond valence parameter $R_{0}$ in the equation, $S_{i j}=\exp \left(\left(R_{0}-R_{i j}\right) / b\right)$ (Brown, 1978), where $R_{i j}$ is the length and $S_{i j}$ is the valence of the bond between atoms $i$ and $j ; R_{0}$ and $b$ are the empirically determined bond valence parameters, whose values are compiled in crystallographic databases. Note that $R_{0}$ has the unit of bond distances and has unique values for a given cation and anion pair.

The $s$ and $p$ orbital radii refers to the Zunger's pseudopotential core radii sum (Zunger and Cohen, 1979), which, in sharp contrast to the empirical $R_{0}$ parameter, is derived directly from quantummechanical calculations. They refer to the core distance of the wave function at which the pseudopotential crosses zero for a given angular momentum of an orbital. Here, we considered $s$ and p-orbitals for our classification. In Table 1, we list all features that were considered in this study.

We started with the $\mathrm{ABX}_{3}$ perovskite halide formability dataset used by Li et al. (2008) consisting of 186 labeled compounds. From this dataset, five compounds (viz., $\mathrm{KSmCl}_{3}, \mathrm{CsGeCl}_{3}, \mathrm{LiCoBr}_{3}$, $\mathrm{KCoBr}_{3}$, and $\mathrm{KCoI}_{3}$ ) were omitted since the bond valence features were not available for these compounds. Furthermore, we augmented the dataset with four tin fluorides, namely $\mathrm{NaSnF}_{3}$, $\mathrm{KSnF}_{3}, \mathrm{RbSnF}_{3}$, and $\mathrm{CsSnF}_{3}$, for which formability labels became available only recently (Tran and Halasyamani, 2014). Thus, the final dataset contained $185 \mathrm{ABX}_{3}$ compounds with known labels and 11 features.

The chemical space covered by the compounds in the training dataset is depicted in Figure 1B. The $185 \mathrm{ABX}_{3}$ perovskite halides contain eight different A-site cations (viz. Ag, Cs, Cu, K, Li, Na, $\mathrm{Rb}$, and $\mathrm{Tl}$ ) and twenty $\mathrm{B}$-site cations (viz. $\mathrm{Ba}, \mathrm{Be}, \mathrm{Ca}, \mathrm{Cd}, \mathrm{Co}$, $\mathrm{Cr}, \mathrm{Cu}, \mathrm{Eu}, \mathrm{Fe}, \mathrm{Hg}, \mathrm{Mg}, \mathrm{Mn}, \mathrm{Ni}, \mathrm{Pb}, \mathrm{Sn}, \mathrm{Sr}, \mathrm{Ti}, \mathrm{V}, \mathrm{Zn}$, and $\mathrm{Zr}$ ). $\mathrm{Cu}$ appears on either $\mathrm{A}$ - or B-sites. The $\mathrm{X}$-site has four different possible choices of $\mathrm{F}, \mathrm{Cl}, \mathrm{Br}$, and $\mathrm{I}$.

Owing to the inherent interpolative nature of ML approaches, we confined our exploration to the above restricted chemical space of perovskite halides throughout this study. Within this space, a total of 640 unique compounds exist, out of which $185(<30 \%)$ are known from previous experiments. The remaining 455 are not explored in the literature and our objective is learned from 185 known compounds via ML and infer or predict the formability of the remaining 455 compositions for rationally guiding the experimental synthesis efforts.

\section{MACHINE LEARNING MODEL}

For the binary classification problem at hand, each instance of our data is described by an $\Omega$-dimensional feature vector $\vec{x}=\left(f_{1}, f_{2}, f_{3}, \ldots, f_{\Omega}\right)$ and a label $y$. The label has a value of
TABLE 1 | A summary of various geometric and electronic properties used in constructing features for the binary classification

\begin{tabular}{ll}
\hline Symbols & Property description \\
\hline$r_{A}^{i}, r_{B}^{i}$, and $r_{X}^{i}$ & Shannon's ionic radii for the A-, B-, and X-site atoms \\
$t_{f}$ & Tolerance factor defined as $\frac{r_{A}^{i}+r_{X}^{i}}{\sqrt{2}\left(r_{B}^{i}+r_{X}^{i}\right)}$ \\
$O_{f}$ & Octahedral factor defined as $\frac{r_{B}^{i}}{r_{X}^{i}}$ \\
$r_{A-X}^{b}$ & Bond valence radii for A-X \\
$r_{B-X}^{b}$ & and B-X bonds \\
$\Delta \chi_{A B-X}^{M B}$ & Differences in the Martynov-Batsanov \\
$\Delta \chi_{B-X}^{M B}$ & electronegativity scales for A-X and B-X atoms \\
$r_{A}^{s+p}, r_{B}^{s+p}$, and $r_{X}^{S+p}$ & Sums of the $s$ and $p$ orbital radii for the A, B, and $X$ atoms
\end{tabular}

Feature ID: feature expression

\begin{tabular}{lll}
\hline 1: $r_{A}^{i}$ & $2: r_{B}^{i}$ & $3: r_{X}^{i}$ \\
$4: t_{f}$ & $5: o_{f}$ & $6: r_{A-X}^{b}$ \\
$7: r_{B-X}^{b}$ & 8: $\left(r_{A}^{i} / r_{X}^{i}\right) \Delta \chi_{A-X}^{M B}$ & $9:\left(r_{B}^{i} / r_{X}^{i}\right) \Delta \chi_{B-X}^{M B}$ \\
10: $\frac{r_{A}^{S+p}}{r_{X}^{S+p}}$ & 11: $\frac{s_{B}^{B}}{r_{X}^{S+p}}$ & \\
\hline
\end{tabular}

+1 , say for perovskites, and -1 , for non-perovskites. A support vector machine aims to find a function that for any given $\vec{x}$ has a value of \pm 1 . Ideally, it is desired to generate a decision boundary in the space of features that maximizes the distance (also known as margin) of the closest instance from either class from it. Instances are defined as points in the hyperspace of features that lie on one side or the other of this hypersurface.

Often a clear separation of the data via a finite margin is not possible. In such cases, a soft margin support vector machine is constructed instead. This classifier allows misclassification of instances; in other words, points in the margin are allowed. If we represent our input data by the set of labeled instances $\left\{\left(\vec{x}_{i}, y_{i}\right)\right\}$, then a soft margin support vector classifier determines the hypersurface in the space of features by solving

$$
\alpha_{1}^{*}, \ldots, \alpha_{n}^{*}=\underset{\alpha_{1}, \ldots, \alpha_{m}}{\arg \min }-\frac{1}{2} \sum_{i=1}^{m} \sum_{j=1}^{m} \alpha_{i} \alpha_{j} \mathcal{K}\left(\vec{x}_{i}, \vec{x}_{j}\right)+\sum_{i=1}^{m} \alpha_{i}
$$

subjected to the following constraints:

$$
0 \leq \alpha_{i} \leq C \quad \text { and } \quad \sum_{i=1}^{m} \alpha_{i} y_{i}=0
$$

Here, the parameter $C$ controls the number of misclassifications. In the minimization, the competition is between the size of the margin and the degree of misclassification acceptable. The support vectors are identified as those points for which $0<\alpha_{i}<C$.

The term $\mathcal{K}\left(\vec{x}_{i}, \vec{x}_{j}\right)$ is known as the kernel, for which there are several choices, for example, linear kernels, polynomial kernels, or Gaussian kernels (also known as radial basis functions or RBFs) (Pedregosa et al., 2011). If the kernel is linear, the decision boundary is always a hyperplane. With two features, the linear 
kernel support vector machine draws a straight line through the data and, hence, is analogous to drawing a structure map. This was another reason that we choose support vector machine over other classification methods as a linear kernel with just two features mimics what has been done in the past.

In this work, however, after testing a number of kernels for their classification accuracies, we chose to go forward with a Gaussian kernel, defined as:

$$
\mathcal{K}\left(\vec{x}_{i}, \vec{x}_{j}\right)=\exp \left(-\gamma\left|\vec{x}_{i}-\vec{x}_{j}\right|^{2}\right) .
$$

To use a support vector machine as a classifier, we first need to select a kernel and set its parameters. For the RBF kernel, the number of parameters that need to be set are two, namely $C$ and $\gamma$. To aid in selecting these parameters, we used gridsearch cross-validation (Pedregosa et al., 2011) that generates a two-dimensional grid. For each grid point, we used five-fold crossvalidation on a $0.8 / 0.2$ training/testing split of the dataset. Our metric of success is the accuracy, that is, the number of instances in the test set predicted correctly divided by the total number of instances in the test set. For this metric, the grid for $C$ often had a number of points with nearly identical values. In many cases, repeating the analysis with a different random number sequence produced variations nearly comparable. For $\gamma$, the best results were obtained when the parameter was varied inversely with number of features; i.e., when $\gamma \propto 1 / \Omega$. Instead of choosing the parameter values at the grid point with the best value of the metric for a given kernel, we simply choose $C=1$ and $\gamma=1 / \Omega$ to define the model. With these parameters, we tested all possible combination of the features going up to four features. These results are discussed in the next section.

\section{RESULTS AND DISCUSSION}

Using the SVC model, we tested all possible models built by taking two $\left({ }^{11} \mathrm{C}_{2}=55\right.$ models with $\left.\Omega=2\right)$, three $\left({ }^{11} \mathrm{C}_{3}=165\right.$ models with $\Omega=3$ ), and four features at a time $\left({ }^{11} \mathrm{C}_{4}=330\right.$ models with $\Omega=4$ ). Performances of these models were ranked by evaluating their prediction accuracy (i.e., fraction of the correctly predicted formability labels) on a $20 \%$ independent test set that was not used for model training. Performance of the top- 5 models for each of the cases with $\Omega=2,3$, and 4 is summarized in Table 2 and Figure 2. We also tested each model for its stability by evaluating prediction variability over 500 different randomly selected test sets. The SDs on test set prediction accuracies for the top performing models are provided in Table 2 .

The top performing model with $\Omega=2$ is the classical toleranceand octahedral-factor pair $\left(t_{f}, o_{f}\right)$. While $t_{f}$ appears in all five top-performing models with $\Omega=2$, performance of the $t_{f}, o_{f}$ pair was found to be remarkably better than the other models. This pair leads to classification accuracies of $92.5 \%$ on the training set and $91.5 \%$ on the test set. It was also interesting to compare the classification performance of this feature pair when individual features were used with and without normalization. For normalization, we scaled each of the features to have a zero mean and unit variance. Figure 3 compares the results of the structure map. We find that the model with normalized features not only results in superior prediction performance but also leads
TABLE 2 | A summary of top-performing features in SVC models with varying $\Omega$.

\begin{tabular}{lllllll}
\hline$\Omega$ & Feature IDs & \multicolumn{2}{c}{ Training set (80\%) } & & \multicolumn{2}{c}{ Test set (20\%) } \\
\cline { 6 - 7 } & & Accuracy & $\sigma$ & & Accuracy & $\sigma$ \\
\hline 2 & $(4,5)$ & 0.925 & 0.013 & & 0.915 & 0.044 \\
2 & $(4,9)$ & 0.878 & 0.012 & & 0.860 & 0.054 \\
2 & $(4,11)$ & 0.828 & 0.012 & & 0.825 & 0.054 \\
2 & $(4,10)$ & 0.849 & 0.019 & & 0.824 & 0.066 \\
2 & $(4,8)$ & 0.843 & 0.016 & & 0.823 & 0.064 \\
3 & $(1,2,5)$ & 0.935 & 0.014 & & 0.914 & 0.046 \\
3 & $(2,4,5)$ & 0.928 & 0.011 & & 0.912 & 0.046 \\
3 & $(2,4,6)$ & 0.921 & 0.011 & & 0.902 & 0.044 \\
3 & $(1,4,5)$ & 0.926 & 0.013 & & 0.901 & 0.048 \\
3 & $(4,5,9)$ & 0.912 & 0.010 & & 0.901 & 0.047 \\
4 & $(1,2,4,5)$ & 0.938 & 0.011 & & 0.921 & 0.042 \\
4 & $(1,2,5,9)$ & 0.927 & 0.013 & & 0.907 & 0.046 \\
4 & $(2,4,5,6)$ & 0.918 & 0.012 & & 0.902 & 0.046 \\
4 & $(1,2,4,9)$ & 0.921 & 0.012 & & 0.901 & 0.043 \\
4 & $(1,2,5,11)$ & 0.924 & 0.014 & 0.896 & 0.052
\end{tabular}

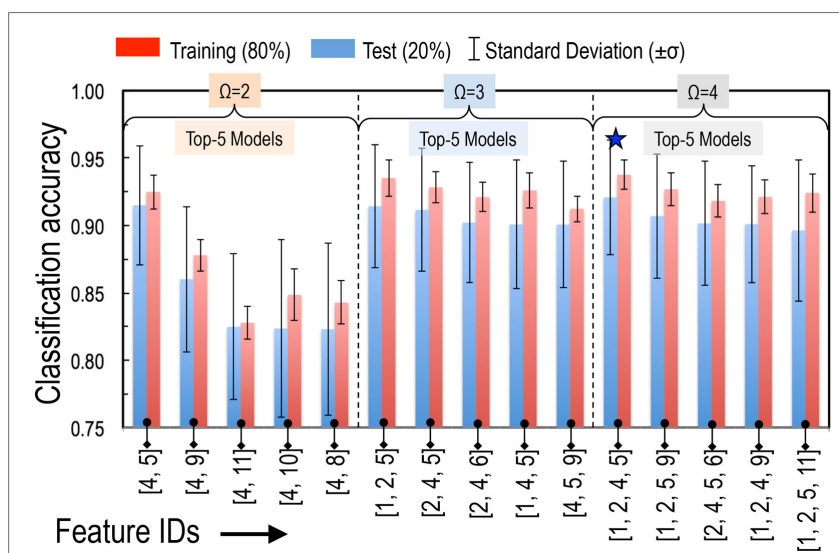

FIGURE 2 | Performance of top-5 models for each of the cases with two features $(\Omega=2)$, three features $(\Omega=3)$, and four features $(\Omega=4)$. Models were ranked by their prediction accuracy defined as fraction of the correctly predicted formability labels on a $20 \%$ test set that was not used for model training. To obtain reliable statistics of our model performance, we performed 500 such trials of randomly choosing $80 \%$ for training and $20 \%$ for testing. At the end of each trial, we have an estimate for the accuracy of the model performance on each test set. Error bars represent the SD $(\sigma)$ of those 500 accuracy estimates. The best performing model is identified with a $\star$.

to a more physically meaningful finite formability region for perovskites. In light of this result, we used normalized features for all SVC models in our study.

The models with $\Omega=3$ and $\Omega=4$ provide only slight improvements in the prediction accuracies. For example, our best performing model with $\Omega=4$ (i.e., with features $r_{A}^{i}, r_{B}^{i}, t_{f}$, and $o_{f}$ ) led to improvements to both the training set and test set prediction accuracies by about $1 \%$. We were able to classify training and test sets formabilities with 93.8 and $92.1 \%$ accuracies, respectively. Ninety-five percent confidence intervals (i.e., $\pm 1.96 \sigma$ ) on these predictions were within 4.3 and $16.5 \%$, respectively. Going beyond $\Omega=4$ did not improve the prediction accuracies and, therefore, we used our best performing model with the four features for subsequent analysis and to make formability predictions on the 
A

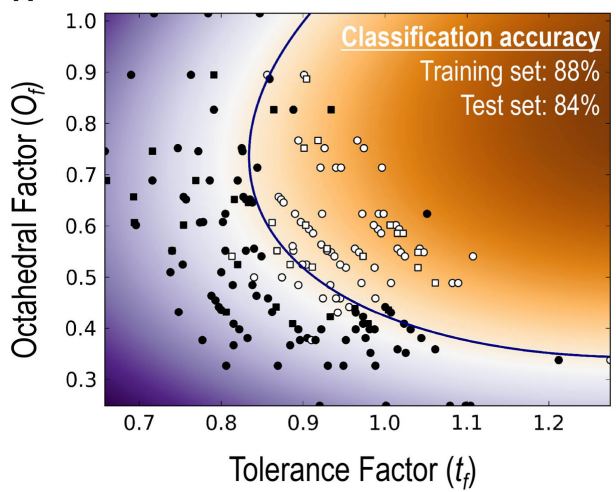

B

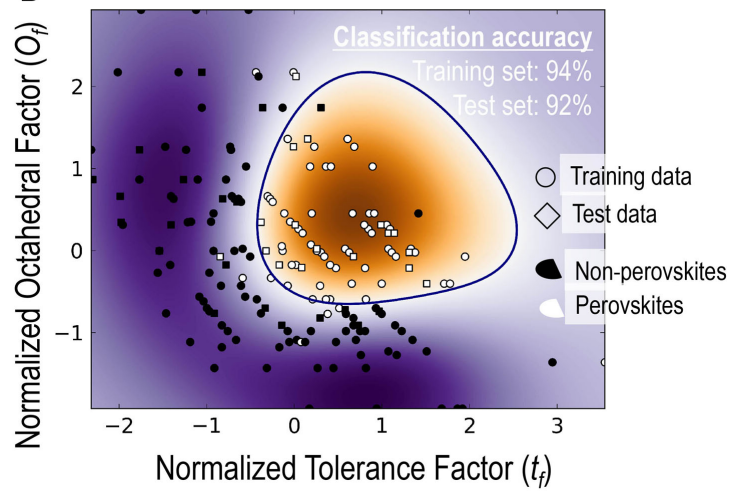

FIGURE 3 | A comparison of classification performance for the SVC models with $t_{f}-O_{f}$ feature pair (A) when both features used as is and (B) when both features were normalized with a zero mean and unit variance.

compounds in the target chemical space which have not yet been synthesized.

Before moving on to new predictions, we also analyzed compounds that were misclassified. The results are presented in Figure 4. While all four features were used in the classification model, here we plot the results in $t_{f}$ and $o_{f}$ feature space for visualization purposes. It can be seen that most of the misclassifications occur at the boundary of the perovskite and nonperovskite regions. Furthermore, model-predicted probabilities of formation for most of these compounds were close to $50 \%$ for both the classes. For instance, the predicted probabilities of $\mathrm{RbEuCl}_{3}$ and $\mathrm{RbSrCl}_{3}$ for being a perovskite were 58 and $52 \%$, respectively. Given that many non-perovskite oxides can be synthesized in a long-lived metastable perovskite phase through non-equilibrium high pressure synthesis routes, it will not be unreasonable to contemplate that such possibilities may also exist for these borderline halide $\mathrm{ABX}_{3}$ chemistries as well. Finally, a compound that comes forth as clear exception is $\mathrm{CsMnF}_{3}$, which was labeled as non-perovskite but predicted to be a perovskite with a $96 \%$ probability. Not surprisingly, we found that that the hexagonal antiferromagnetic structure of $\mathrm{CsMnF}_{3}$ can be easily transformed to cubic perovskite at high pressures (Kafalas and Longo, 1972). Therefore, such misclassifications should be looked at, not so much as learning model failures, but rather as indicators of possibilities for alternative synthesis routes (such as high temperature, pressure, or epitaxial strain) toward perovskite crystal structures (Balachandran et al., 2015).

Having demonstrated classification using known data, we now use the ML model to predict formability (i.e., perovskite vs. non-perovskite) of the unlabeled $\mathrm{ABX}_{3}$ chemistries. While, in principle, we were able to classify all of the 455 chemical compositions, going forward, we focus our attention on the top-40 $\mathrm{ABX}_{3}$ chemistries, all of which were classified as a perovskite with a probability $\geq 85 \%$. These systems are listed in Table 3 along with the predicted probability of formation in a perovskite structure. A complete list of predictions on the entire dataset of 455 compounds can be found in Supplementary Material. It is interesting to note that some of these compounds [ such as $\mathrm{TlCaF}_{3}$ and $\mathrm{TlHgF}_{3}$ have also been predicted to be stable in the perovskite crystal structure by a recent independent study (Körbel et al., 2016)].

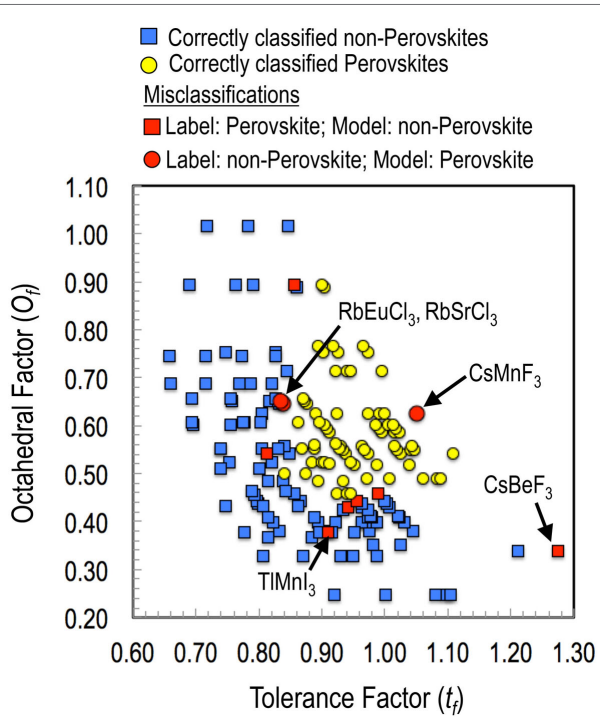

FIGURE 4 | Analysis of the training set misclassifications for the best performing model with $\Omega=4$ (i.e., with features $r_{A}^{i}, r_{B}^{i}, t_{f}$, and $o_{f}$ ). While all of the four features were used in the classification model; here, the results are plotted in a two-dimensional plot with the $t_{f}$ and the $o_{f}$ for better intelligibility.

Finally, we note that our ML-based formability prediction model has only considered structural factors and other easily accessible attributes for the $\mathrm{ABX}_{3}$ systems. This is a reasonable first screening step that allows us to efficiently down-select a small fraction of the overall unlabeled dataset (i.e., 40 out of a total of 455 possibilities, viz., $<10 \%)$. However, to make reliable predictions relative thermodynamic stability of the identified $\mathrm{ABX}_{3}$ chemistries has to be rigorously tested against all potential chemical combinations that may combine to form the composition of interest. More specifically, this requires computation of relative stability with respect to a set of most stable known materials (including elemental, binaries, and ternaries chemistries) at that chemical composition for each of the top- $40 \mathrm{ABX}_{3}$ chemistries identified here. Furthermore, one has to confirm the absence of any soft mode instabilities over the entire Brillouin zone in order 
TABLE 3 | Model predicted novel $\mathrm{ABX}_{3}$ chemistries with a probability of $\geq 85 \%$ to form a perovskite structure.

\begin{tabular}{|c|c|c|c|c|c|c|c|c|c|}
\hline System & Probability & System & Probability & System & Probability & System & Probability & System & Probability \\
\hline $\mathrm{TITiF}_{3}$ & 1.00 & $\mathrm{TIZnF}_{3}$ & 0.99 & $\mathrm{RbZrF}_{3}$ & 0.98 & $\mathrm{RbHgCl}_{3}$ & 0.95 & $\mathrm{RbHgBr}_{3}$ & 0.89 \\
\hline $\mathrm{RbTiF}_{3}$ & 1.00 & $\mathrm{KZrF}_{3}$ & 0.99 & $\mathrm{CsCrF}_{3}$ & 0.97 & $\mathrm{TICaCl}_{3}$ & 0.95 & $\mathrm{NaTiF}_{3}$ & 0.87 \\
\hline $\mathrm{KTiF}_{3}$ & 1.00 & $\mathrm{AgTiF}_{3}$ & 0.99 & $\mathrm{CsVF}_{3}$ & 0.97 & $\mathrm{RbNiF}_{3}$ & 0.94 & $\mathrm{CsZrF}_{3}$ & 0.87 \\
\hline $\mathrm{TIVF}_{3}$ & 1.00 & $\mathrm{AgZrF}_{3}$ & 0.99 & $\mathrm{CsCaBr}_{3}$ & 0.97 & $\mathrm{TH}_{\mathrm{HgCl}}$ & 0.93 & $\mathrm{CsCal}_{3}$ & 0.86 \\
\hline $\mathrm{AgVF}_{3}$ & 0.99 & $\mathrm{TICaF}_{3}$ & 0.98 & $\mathrm{TIHgF} 3$ & 0.96 & $\mathrm{CsZnF}_{3}$ & 0.91 & $\mathrm{AgCdF}_{3}$ & 0.86 \\
\hline $\mathrm{AgCrF}_{3}$ & 0.99 & $\mathrm{TIMgF}_{3}$ & 0.98 & $\mathrm{CsFeF}_{3}$ & 0.96 & $\mathrm{RbCaBr}_{3}$ & 0.89 & $\mathrm{TICaBr}_{3}$ & 0.86 \\
\hline $\mathrm{AgFeF}_{3}$ & 0.99 & $\mathrm{TIZrF}_{3}$ & 0.98 & $\mathrm{NaZrF}_{3}$ & 0.96 & $\mathrm{CsCuF}_{3}$ & 0.89 & $\mathrm{CsEuBr}_{3}$ & 0.86 \\
\hline $\mathrm{CsTiF}_{3}$ & 0.99 & $\mathrm{RbMgF}_{3}$ & 0.98 & $\mathrm{TINiF}_{3}$ & 0.95 & $\mathrm{CsHgl}_{3}$ & 0.89 & $\mathrm{RbTiCl}_{3}$ & 0.85 \\
\hline
\end{tabular}

to establish the dynamical stabilities of these systems. Such efforts are currently underway.

\section{CONCLUSION}

Perovskite halides (hybrid and inorganic) have garnered significant interest because of their outstanding photovoltaic properties. One of the outstanding challenges that concern experimental efforts in this direction is in successfully synthesizing phase pure perovskite compounds. We attempted to address this by employing a ML approach, which allows us to rapidly screen and identify candidate $\mathrm{ABX}_{3}$ perovskite compounds for experimental evaluation. From our analysis, we identified a combination of features that best represent the description of a hardsphere model (ionic radii, tolerance factor, and octahedral factors) in classifying perovskites from non-perovskites. The key insight is that, in halides, interactions that govern geometric packing and steric effects are important for classification, in spite of the fact that there were transition metal cations in our training set. However, we anticipate that additional electronic effects, such as crystal-field stabilization energy, might emerge as critical features to accurately describe different octahedral tilt patterns within a perovskite sub-class, which we do not discuss here. Furthermore, the ability to rationally establish decision boundaries and assign uncertainties with predictions and misclassifications makes this approach highly attractive for predictive materials

\section{REFERENCES}

Balachandran, P. V., Theiler, J., Rondinelli, J. M., and Lookman, T. (2015). Materials prediction via classification learning. Sci. Rep. 5, 13285. doi:10.1038/ srep13285

Balachandran, P. V., Xue, D., Theiler, J., Hogden, J., and Lookman, T. (2016). Adaptive strategies for materials design using uncertainties. Sci. Rep. 6, 19660. doi:10.1038/srep19660

Brown, I. D. (1978). Bond valences? A simple structural model for inorganic chemistry. Chem. Soc. Rev. 7, 359. doi:10.1039/CS9780700359

Ceder, G., Hauthier, G., Jain, A., and Ong, S. P. (2011). Recharging lithium battery research with first-principles methods. Mater. Res. Soc. Bull 36, 185. doi:10.1557/ mrs.2011.31

Curtarolo, S., Hart, G. L. W., Nardelli, M. B., Mingo, N., Sanvito, S., and Levy, O. (2013). The high-throughput highway to computational materials design. Nat. Mater. 12, 191. doi: $10.1038 /$ nmat3568

Curtarolo, S., Setyawan, W., Wang, S., Xue, J., Yang, K., Taylor, R. H., et al. (2012). AFLOWLIB.ORG: a distributed materials properties repository from high-throughput ab initio calculations. Comput. Mater. Sci. 58, 227. doi:10.1016/ j.commatsci.2012.02.002

Feng, L., Jiang, L., Zhu, M., Liu, H., Zhou, X., and Li, C. (2008). Formability of $\mathrm{ABO}_{3}$ cubic perovskites. J. Phys. Chem. Solids 69, 967. doi:10.1016/j.jpcs.2007.11.007 design. We demonstrated this by identifying 40 new $\mathrm{ABX}_{3}$ compounds that show potential for forming stable perovskite structure-type and, to the best of our knowledge, have not been reported previously. A detailed study targeted to assess thermodynamic and dynamical stabilities of these compounds is currently underway.

\section{AUTHOR CONTRIBUTIONS}

GP, PB assembled the halide formability dataset and the feature set used in learning. GP performed the machine learning. All authors analyzed the results and contributed in writing the manuscript.

\section{FUNDING}

GP, PB and TL acknowledge support from the Los Alamos National Laboratory (LANL) Laboratory Directed Research and Development (LDRD). Los Alamos National Laboratory is operated by Los Alamos National Security, LLC, for the National Nuclear Security Administration of the (U.S.) Department of Energy under contract DE-AC52-06NA25396.

\section{SUPPLEMENTARY MATERIAL}

The Supplementary Material for this article can be found online at http://journal.frontiersin.org/article/10.3389/fmats.2016.00019

Flach, P. (2012). Machine Learning: The Art and Science of Algorithms that Make Sense of Data. Cambridge: Cambridge University Press.

Jain, A., Hautier, G., Moore, C. J., Ong, S. P., Fischer, C. C., Mueller, T., et al. (2011). A high-throughput infrastructure for density functional theory calculations. Comput. Mater. Sci. 50, 2295. doi:10.1016/j.commatsci.2011.02.023

Kafalas, J. A., and Longo, J. M. (1972). High pressure synthesis of $\left(\mathrm{ABX}_{3}\right)(\mathrm{AX})_{n}$ compounds. J. Solid State Chem. 4, 55. doi:10.1016/0022-4596(72)90132-6

Kim, C., Pilania, G., and Ramprasad, R. (2016). From organized high-throughput data to phenomenological theory using machine learning: the example of dielectric breakdown. Chem. Mater 28, 1304-1311. doi:10.1021/acs.chemmater. 5 b04109

Körbel, S., Marques, M. A. L., and Botti, S. (2016). Stability and electronic properties of new inorganic perovskites from high-throughput ab initio calculations. J. Mater. Chem. C doi:10.1039/c5tc04172d

Kumar, A., Verma, A. S., and Bhardwaj, S. R. (2008). Prediction of formability in perovskite-type oxides. Open Appl. Phys. J. 1, 11. doi:10.2174/ 1874183500801010011

Li, C., Lu, X., Ding, W., Feng, L., Gao, Y., and Guo, Z. (2008). Formability of $\mathrm{ABX}_{3}(\mathrm{X}=\mathrm{F}, \mathrm{Cl}, \mathrm{Br}, \mathrm{I})$ halide perovskites. Acta Cryst. B 64, 702. doi:10.1107/ S0108768108032734

Li, C., Soh, K. C. K., and Wu, P. (2004). Formability of $\mathrm{ABO}_{3}$ perovskites. J. Alloys Compd. 372, 40. doi:10.1016/j.jallcom.2003.10.017 
Mannodi-Kanakkithodi, A., Pilania, G., Huan, T. D., Lookman, T., and Ramprasad, R. (2016). Machine learning strategy for accelerated design of polymer dielectrics. Sci. Rep. 6, 20952. doi:10.1038/srep20952

Mitchell, R. H. (2002). Perovskites: Modern and Ancient. Ontario: Almaz Press.

Mooser, E., and Pearson, W. B. (1959). On the crystal chemistry of normal valence compounds. Acta Cryst. 12, 1015. doi:10.1107/S0365110X59002857

Muller, O., and Roy, R. (1974). The Major Ternary Structural Families. New York: Springer.

Pedregosa, F., Varoquaux, G., Gramfort, A., Michel, V., Thirion, B., Grisel, O., et al. (2011). Scikit-learn: machine learning in Python. J. Mach. Learn. Res. 12, 2825.

Pilania, G., Balachandran, P. V., Gubernatis, J. E., and Lookman, T. (2015). Classification of $\mathrm{ABO}_{3}$ perovskite solids: a machine learning study. Acta Cryst. B 71, 507. doi:10.1107/S2052520615013979

Pilania, G., Gubernatis, J. E., and Lookman, T. (2015a). Structure classification and melting temperature prediction in $\mathrm{AB}$ solids via machine learning. Phys. Rev. $B$ 91, 214302. doi:10.1103/PhysRevB.91.214302

Pilania, G., Gubernatis, J. E., and Lookman, T. (2015b). Classification of octet AB-type binary compounds using dynamical charges: a materials informatics perspective. Sci. Rep. 5, 17504. doi:10.1038/srep17504

Pilania, G., and Lookman, T. (2014). Electronic structure and biaxial strain in $\mathrm{RbHgF}_{3}$ perovskite and hybrid improper ferroelectricity in $(\mathrm{Na}, \mathrm{Rb}) \mathrm{Hg}_{2} \mathrm{~F}_{6}$ and (K,Rb) $\mathrm{Hg}_{2} \mathrm{~F}_{6}$ superlattices. Phys. Rev. B 90, 115121. doi:10.1103/PhysRevB.90. 115121

Pilania, G., Mannodi-Kanakkithodi, A., Gubernatis, J. E., Ramprasad, R., and Lookman, T. (2016). Machine learning bandgaps of double perovskites dielectrics. Sci. Rep. 6, 19375. doi:10.1038/srep19375

Pilania, G., and Uberuaga, B. P. (2015). Cation ordering and effect of biaxial strain in double perovskite CsRbCaZnCl 6 . J. Appl. Phys 117, 114103. doi:10.1063/1. 4915938

Pilania, G., Wang, C., Jiang, X., Rajasekaran, S., and Ramprasad, R. (2013). Accelerating materials property predictions using machine learning. Sci. Rep. 3, 2810. doi:10.1038/srep02810

Rabe, K. M., Phillips, J. C., Villars, P., and Brown, I. D. (1992). Global multinary structural chemistry of stable quasicrystals, high- $\mathrm{T}_{C}$ ferroelectrics, and high-T-c superconductors. Phys. Rev. B 45, 7650. doi:10.1103/PhysRevB.45. 7650

Sarukura, N., Murakami, H., Estacio, E., Ono, S. G., El Ouenzerfi, R., Cadatal, M., et al. (2007). Proposed design principle of fluoride-based materials for deep ultraviolet light emitting devices. Opt. Mater. 30, 15. doi:10.1016/j.optmat.2006. 11.031
Service, R. F. (2012). Materials scientists look to a data-intensive future. Science 335 , 1434. doi:10.1126/science.335.6075.1434

Sharma, V., Wang, C., Lorenzini, R. G., Ma, R., Zhu, Q., Sinkovits, D. W., et al. (2014). Rational design of all organic polymer dielectrics. Nat. Commun. 5, 4845. doi:10.1038/ncomms5845

Tran, T. T., and Halasyamani, P. S. (2014). Effect of $\mathrm{SiO}_{2}, \mathrm{NaCl}, \mathrm{Al}_{2} \mathrm{O}_{3}$, and $\mathrm{FeCl}_{3}$ on phase change behavior of supported and unsupported $\mathrm{TiO}_{2}$. J. Solid State Chem. 210, 213. doi:10.1006/jssc.1993.1282

Vapnik, V. (1995). The Nature of Statistical Learning Theory. New York: Springer.

Vapnik, V. (1998). Statistical Learning Theory. New York: John Wiley and Sons.

Computational Materials Repository. (2015). Available at: https://wiki.fysik.dtu.dk/ $\mathrm{cmr} /($ Documentation); https://cmr.fysik.dtu.dk/

Materials Project - A Materials Genome Approach. (2015). Available at: http:// materialsproject.org/

Yu, L., and Zunger, A. (2012). Identification of potential photovoltaic absorbers based on first-principles spectroscopic screening of materials. Phys. Rev. Lett. 108, 068701. doi:10.1103/PhysRevLett.108.068701

Zhang, F., Mao, Y., Park, T.-J., and Wong, S. S. (2008). Green synthesis and property characterization of single-crystalline perovskite fluoride nanorods. Adv. Funct. Mater. 18, 103. doi:10.1002/adfm.200700655

Zhang, H., Li, N., Li, K., and Xue, D. (2007). Structural stability and formability of $\mathrm{ABO}_{3}$-type perovskite compounds. Acta Cryst. B 63, 812. doi:10.1107/ S0108768107046174

Zunger, A., and Cohen, M. L. (1979). First-principles nonlocal-pseudopotential approach in the density-functional formalism. II. Application to electronic and structural properties of solids. Phys. Rev. B 20, 4082. doi:10.1103/PhysRevB.20. 4082

Conflict of Interest Statement: The authors declare that the research was conducted in the absence of any commercial or financial relationships that could be construed as a potential conflict of interest.

The reviewer XW and handling Editor declared their shared affiliation, and the handling Editor states that the process nevertheless met the standards of a fair and objective review.

Copyright (C) 2016 Pilania, Balachandran, Kim and Lookman. This is an open-access article distributed under the terms of the Creative Commons Attribution License (CC $B Y)$. The use, distribution or reproduction in other forums is permitted, provided the original author(s) or licensor are credited and that the original publication in this journal is cited, in accordance with accepted academic practice. No use, distribution or reproduction is permitted which does not comply with these terms. 